Cite as: Janacsek, K., Ambrus, G. G., Paulus, W., Antal, A., \& Nemeth, D. (2015). Right hemisphere advantage in statistical learning: evidence from a probabilistic sequence learning task. Brain stimulation, 8(2), 277-282.

\title{
Right hemisphere advantage in statistical learning: Evidence from a probabilistic sequence
}

\section{learning task}

Karolina Janacsek ${ }^{1,2^{*}}$, Geza Gergely Ambrus ${ }^{3,4^{*}}$, Walter Paulus ${ }^{3}$, Andrea Antal ${ }^{3 *}$, Dezso Nemeth $^{1,5^{*}}$

*These authors contributed equally to this work.

${ }^{1}$ Department of Clinical Psychology and Addiction, Eotvos Lorand University, Budapest, Hungary

${ }^{2}$ Department of Psychology, Georgetown University, Washington DC, USA

${ }^{3}$ Department of Clinical Neurophysiology, Georg-August University, Göttingen, Germany

${ }^{4}$ Institute of Medical Psychology and Medical Sociology, University Medical Center, GeorgAugust University, Göttingen, Germany

${ }^{5}$ Imaging Research Center, University of Texas, Austin, USA

Running head: Statistical learning and consolidation in the PFC

Corresponding author: Dezso Nemeth, Ph.D., Associate Professor of Psychology, Imaging Research Center, University of Texas, Austin, USA, and Department of Clinical Psychology and Addiction, Eotvos Lorand University, Budapest, Hungary, H-1064 Budapest, Izabella street 46. Email: nemethd@gmail.com 


\begin{abstract}
Picking up statistical regularities of patterns from the environment is essential for predictive and adaptive behavior. One of the most important challenges is to understand how statistical learning occurs and how the acquired information consolidates and stabilizes in the brain. Evidence suggests that the prefrontal cortex (PFC) has a critical role in these processes; the division of labor between hemispheres, however, is less characterized. The aim of the present study was to directly investigate the causal role of the right and left PFC in statistical learning and its consolidation. Healthy, young adults were trained on a probabilistic sequence learning task. Anodal transcranial direct current stimulation (tDCS) over the right or left dorsolateral PFC (DLPFC) was applied during the training in order to modify learning-related cortical plasticity in the targeted brain regions by increasing neural excitability. Performance was tested during and immediately after the stimulation, 2-hour and 24-hour later. We found that the anodal tDCS over the right DLPFC led to enhanced learning performance both after the 2-hour and 24-hour retention periods, suggesting the causal role of this area in statistical learning. In contrast, we did not find any effect of left DLPFC stimulation on learning. These results highlight the role of the right fronto-striatal network in statistical learning and its consolidation.
\end{abstract}

Keywords: statistical learning, probabilistic sequence learning, implicit learning, prefrontal cortex (PFC), fronto-striatal network, memory consolidation 
During every moment of our life, the brain is picking up statistical regularities of patterns of our physical and social world in order to successfully interact and adapt. The computational underpinnings of these processes are relatively well characterized [1-5], whereas the question of how the brain learns based on these statistical regularities and how it optimizes, stabilizes and

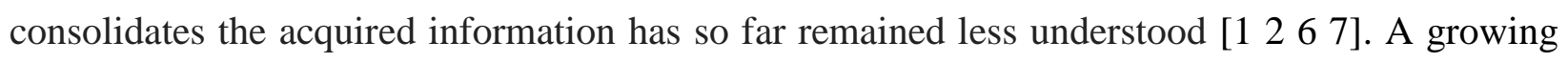
body of evidence suggests that this fundamental learning mechanism relies primarily on frontostriatal brain networks [8-11].

Some studies suggested a hemispheric asymmetry in the role of prefrontal cortex (PFC) in statistical learning. Gazzaniga and colleagues [ $\left.\begin{array}{lll}7 & 12 & 13\end{array}\right]$ used probabilistic guessing and visual statistical learning tasks where some events were more frequent than others. In these tasks a maximizing strategy (picking always the most frequent option) can lead to a better performance compared to the frequency-matching strategy (where people pick the particular options with the approximate frequency of their occurrences). Gazzaniga et al. showed in split-brain and frontal lobe-lesioned patients that the left hemisphere, especially the PFC, is involved in using frequency-matching strategies, whereas the right hemisphere has frequency-maximizing strategies. The advantage of the implemented strategies may depend on the task itself: the frequency matching strategies can be helpful for uncovering simple causal relationships, however they can lead to non-optimal behavior when the relations are more complex (such as in an environment/task with probabilistic statistical features) [13]. Supporting this idea, patients with a left frontal lobe/PFC lesion performed well in the guessing tasks and in statistical learning of new visual features, while the right frontal lobe/PFC lesion led to a weaker performance.

In the present study, we used the Alternating Serial Reaction Time (ASRT) Task [14], which is a unique tool to investigate the processes of frequency-maximizing strategy. In the 
ASRT task, participants are asked to respond to stimuli (Fig. 1A), which appear according to a probabilistic sequence structure (e.g., 2r1r3r4r, where numbers represent specific locations on the screen determined by the sequence, and $\mathrm{r}$ represent randomly selected location). Because of the probabilistic nature of the task, some runs of three consecutive stimuli (i.e. triplets) are more frequent than others. Subjects are sensitive to these regularities and with practice they become better in differentiating between high- and low-frequency events (Fig. 1B). Since there are only two triplet frequencies, namely high-frequency triplets, occurring $62.5 \%$ of all trials, and lowfrequency triplets, occurring $37.5 \%$ of all trials, this type of statistical learning in the ASRT task is based on frequency-maximizing strategy. 


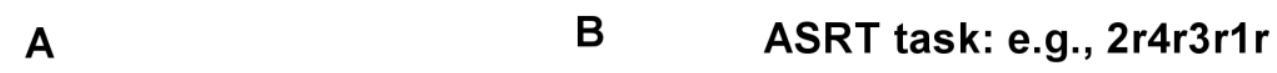

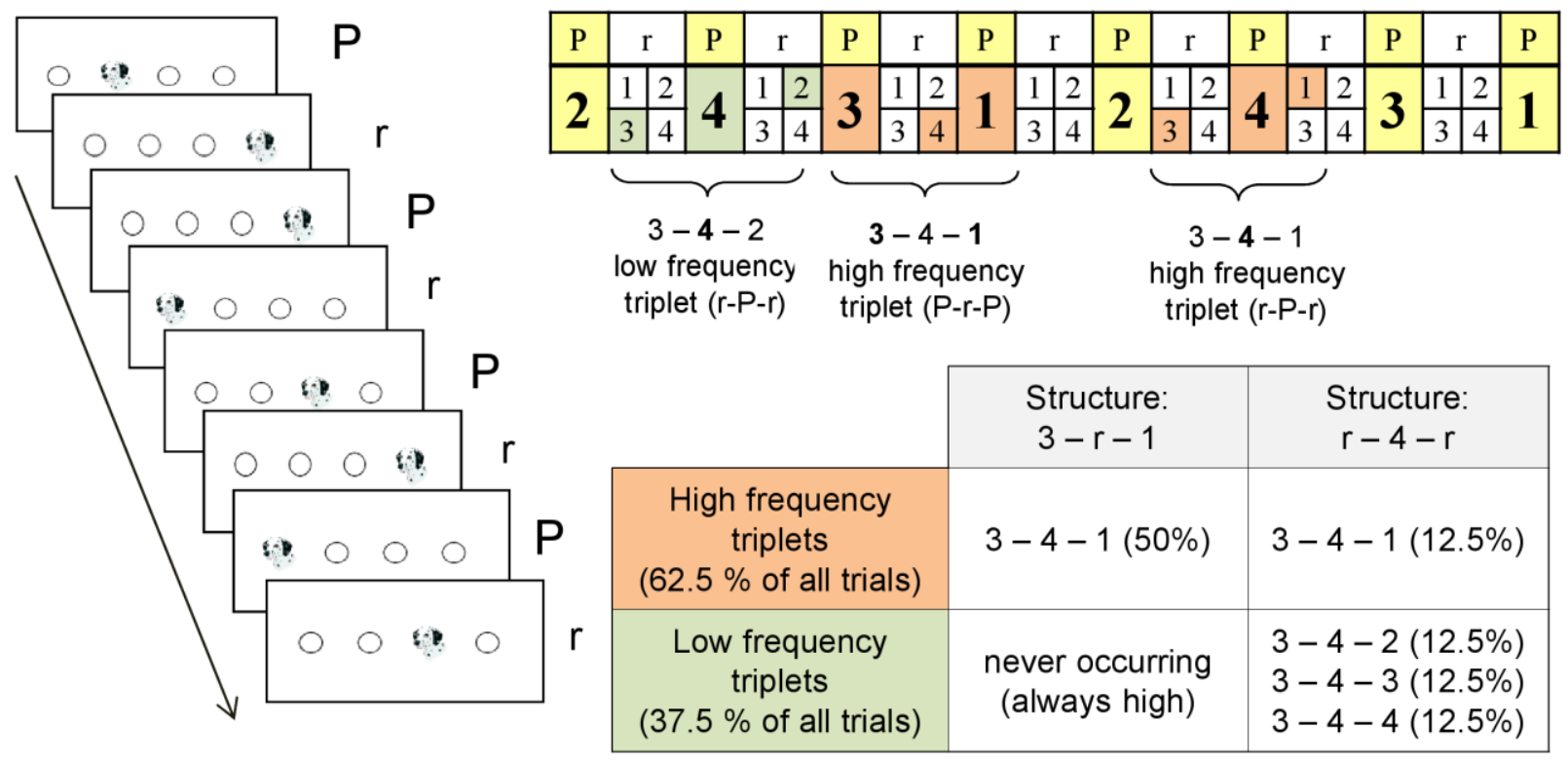

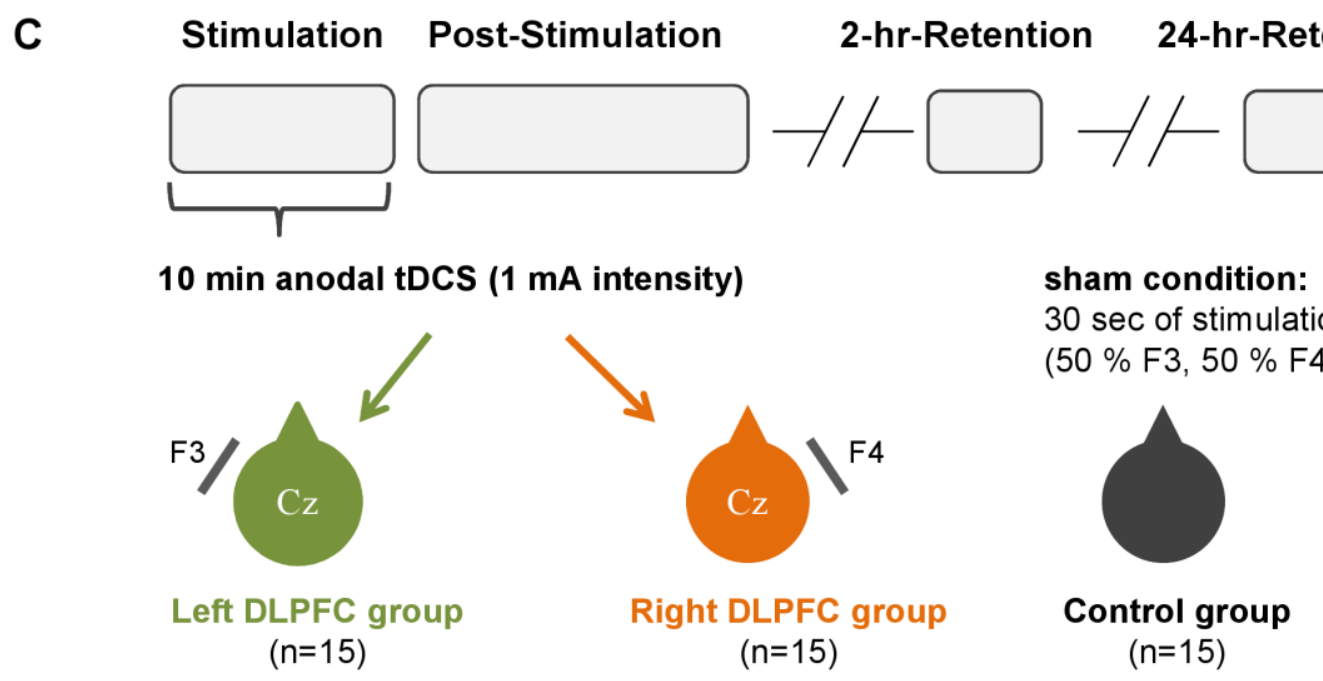

Figure 1. (A) Stimuli of the Alternating Serial Reaction Time (ASRT) task (P - pattern, $r$ - random). (B) Because of the probabilistic nature of the task, some runs of three consecutive stimuli (triplets) occur more frequently than others. For example, if the sequence is $2 \mathrm{r} 4 \mathrm{r} 3 \mathrm{r} 1 \mathrm{r}$, triplets $2-_{-}-4,4-{ }_{-}-3,3-{ }_{-}-1$, and 1-_-2 occur often because the third element could be derived from the sequence or could also be a random element. In contrast, 2-_-3 or 1-_-4 would occur infrequently because in this case the third element could only be random. We refer to the former as highfrequency triplets and the latter as low-frequency triplets. Learning is defined as faster responses to the highfrequency triplets than to the low-frequency ones. (C) Design and procedure of the experiment. Anodal transcranial direct current stimulation (tDCS) was administered simultaneously with the Alternating Serial Reaction Time 
(ASRT) task during the Stimulation Phase. Then - with no delay - participants performed the ASRT task without stimulation (Post-Stimulation Phase). Performance was retested 2-hr and 24-hr later (Retention Phases).

To sum up, several studies [ $\left.\begin{array}{lll}7 & 12 & 13\end{array}\right]$ support the role of the PFC in the mechanisms of picking up statistical relationships and patterns of events in the environment. Furthermore, they highlight that the right and left hemispheres, play a different role in these processes. Here we aimed to go beyond these studies by examining the role of the right vs. left PFC in statistical learning as well as in the consolidation of the acquired information in a healthy, young population. Transcranial direct current stimulation (tDCS) over the right or left dorsolateral PFC (DLPFC) was applied during a probabilistic sequence learning task, namely ASRT, in order to directly investigate the causal role of the targeted areas in the acquisition and consolidation of statistical information. Therefore, performance was tested after 2- and 24-hour retention periods. We hypothesized that the right DLPFC stimulation leads to better statistical learning performance compared to the left DLPFC stimulation and the sham condition because of the underlying triplet structure of the ASRT task.

\section{Material and Methods}

\section{Participants}

Forty-five right-handed young adults participated in the experiment (mean age $=26.18$ years, $\mathrm{SD}=3.91 ;$ mean education $=18.37$ years, $\mathrm{SD}=2.96 ; 24$ males $/ 21$ females $)$. They were randomly assigned to the 1) sham/control $(n=15), 2)$ the left DLPFC stimulation $(n=15)$, or 3) the right DLPFC stimulation ( $\mathrm{n}=15)$ group. Participants had no previous history of neurological or psychiatric disorders, and they had no metal implants in the head or neck area. Participants gave written and verbal informed consent before participating. The experiment was in accordance 
with the guidelines of the Declaration of Helsinki, and was approved by the ethics committee of the University of Göttingen.

\section{Task}

The Alternating Serial Reaction Time (ASRT) Task [14 15] was used to measure probabilistic sequence learning. In this task, a stimulus (a dog's head) appeared in one of the four empty circles on the screen and participants had to press the corresponding button as fast and as accurately as possible (Fig. 1A). The target remained on the screen until the participant pressed the correct button. The response to stimulus interval (RSI) was $120 \mathrm{msec}$. The computer was equipped with a special keyboard which had four marked keys (Y, C, B and M on a German keyboard, equivalent to $\mathrm{Z}, \mathrm{C}, \mathrm{B}$ and $\mathrm{M}$ on a US keyboard), each corresponding to one of the circles [16].

The ASRT task consisted of 35 presentation blocks in total, with 85 stimulus presentations and responses (key presses) per block. The first five stimulus presentations were random for practice purposes, then an eight-element alternating sequence (e.g., 2r4r3r1r, where numbers represent the four places on the screen, and $\mathrm{r}$ represents an event randomly selected from the four possible places) was repeated ten times. Ten presentation blocks of the ASRT task were administered in the Stimulation Phase, 15 presentation blocks in the Post-Stimulation Learning Phase, five presentation blocks in the 2-hr- and also five presentation blocks in the 24hr-Retention Phase (Fig. 1C).

\section{Transcranial direct current stimulation (tDCS)}


Anodal tDCS was used to modify learning-related cortical plasticity in the targeted brain regions (by increasing neural excitability), and thus to identify the causal role of those areas and the related networks in specific behavioral functions. Based on the standard stimulation protocol, a direct current of $1 \mathrm{~mA}$ amplitude for a 10 minutes duration was provided by a NeuroConn (Ilmenau, Germany) stimulator [18]. The stimulation was delivered by two rubber electrodes placed in saline-soaked sponges cut to $5 \times 7 \mathrm{~cm}$ (with the longer axis placed in the anteriorposterior direction) and fixed in place by two rubber bands fastened around the participant's head. The international 10-20 EEG standard was used to locate $\mathrm{Cz}$ on each participant. The anodal electrode was placed over the F3 or F4 for left and right DLPFC stimulation respectively, while the cathode was placed over the $\mathrm{Cz}$ (Fig. 1C). This method to target DLPFC has been used in previous studies [e.g., 19 20] and has been confirmed as an appropriate method of localization by neuronavigation techniques [21]. The current was ramped up to maximum at the beginning over 30 seconds, remained at $1 \mathrm{~mA}$ for 600 seconds (real stimulation conditions), and then ramped down to $0 \mathrm{~mA}$ over 30 seconds. The stimulation set-up remained fixed on the participant's head throughout the task. Following the protocol of Ambrus et al.'s study [18], a 30 seconds long stimulation (with an additional 30-s ramp-up and a 30-s ramp-down period) was used in the control (sham) group in order to blind the participants about whether they were assigned to the experimental (real stimulation) or to the control group (single-blinded design). A recent resting state functional magnetic resonance imaging (fMRI) study has shown that a short (e.g., 30 seconds) tDCS protocol for the sham condition does not have a significant effect on brain dynamics compared to the active tDCS [22], proving it to be an appropriate control condition for investigating the effect of stimulation. 
The Stanford Sleepiness Scale [SSS, 23] was administered before the Stimulation, after the Post-Stimulation, and before and after the 2-hr- and 24-hr Retention Phases in order to assess the potential immediate and after-effects of the stimulation on subjective sleepiness. Participants reported their subjective sleepiness on a 7-point Likert scale where 1 means "Feeling active, vital, alert, or wide awake" and 7 means "No longer fighting sleep, sleep onset soon; having dream-like thoughts".

\section{Statistical analysis}

During the analysis we followed the protocol of previous ASRT studies [e.g., 214 1517], and because of the typically high accuracy in performance in the ASRT task (above 92\%), we focused on reaction time (RT) analysis. We calculated median RTs for correct responses only, for each subject and each block of five (e.g., Blocks 1-5, Blocks 6-10, etc.), for high- and low-frequency triplets separately. Then we calculated a triplet learning index by subtracting the RTs for the high-frequency triplets from the RTs for the low-frequency ones for each block of five. Here, a higher index reflects better statistical learning; thus being faster on high- than on low-frequency triplets. In the analysis reported below, we averaged triplet learning indices across blocks of five, separately for each session of the experiment (Stimulation, Post-Stimulation, 2-hrand 24-hr-Retention Phase).

Note that for each response (n), we defined whether it was a high- or a low-frequency triplet by considering whether it was more or less predictable from the event $n-2$. Two kinds of low-frequency triplets, repetitions (e.g., 222, 333) and trills (e.g., 212, 343), were excluded from the analysis, since people often showed pre-existing response tendencies to them [14 24]. By 
eliminating these repetitions and trills we attempted to ensure that any low- versus highfrequency differences are due to learning and not to preexisting tendencies.

\section{Results}

We conducted a mixed design analysis of variance (ANOVA) on triplet learning indices shown on Figure 2 with Session (4: Stimulation, Post-Stimulation, 2-hr- and 24-hr-Retention Phase) as a within-subject factor, and GROUP (3: sham, left vs. right DLPFC stimulation) as a between-subject factor. ANOVA revealed significant learning (Intercept: $F(1,42)=164.90, \eta_{\mathrm{p}}^{2}$ $=.80, p<.001)$ which improved with practice (indicated by the main effect of SESSION: $F(3$, $40)=5.74, \eta^{2}=.30, p=.002$ ). Groups differed in the amount of learning (shown by the main effect of GROUP: $\left.F(2,42)=4.05, \eta^{2}{ }_{\mathrm{p}}=.16, p=.025\right)$. Fisher's LSD pairwise comparisons revealed that the right DLPFC stimulation group exhibited the highest learning performance, differing significantly from the control/sham group ( $p=.008$, Cohen's $d=1.01)$ and on a trend level from the left DLPFC stimulation group ( $p=.073, d=0.65)$. This difference was more evident by the 2-hr- and the 24-hr-Retention Phases, where the Fisher's LSD pairwise comparisons revealed the right DLPFC stimulation group's advantage compared to the control (2-hr-delay: $p=.020, d=0.91 ; 24$-hr-delay: $p=.017, d=0.90$ ) and the left DLPFC stimulation group (24-hr-delay: $p=.012, d=0.80$ ). There were no group differences in learning in the Stimulation and Post-Stimulation Phases ( $p s>.270)$. 


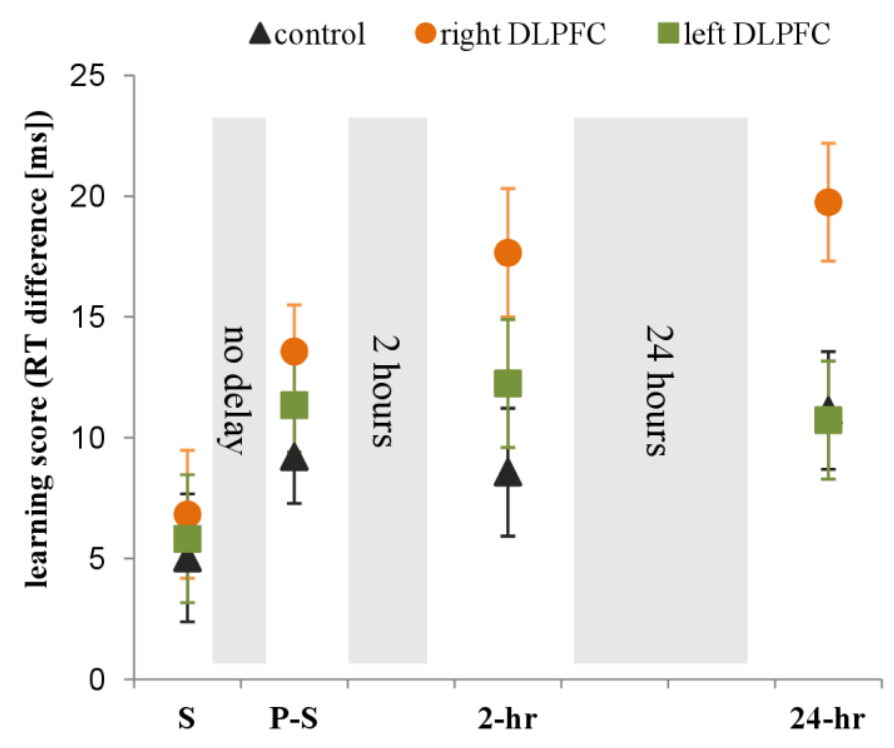

Figure 2. Learning performance (measured as faster responses for high- compared to low-frequency triplets) in the Stimulation (S), Post-Stimulation (P-S), 2-hr- and 24-hr-Retention Phases. Error bars indicate Standard Error of the Mean (SEM).

The observed group differences were not due to a general effect of the stimulation on arousal level since all groups responded with similar RTs in general, in all sessions ( $p$ s > .737). In addition, univariate ANOVAs conducted on Stanford Sleepiness measures with GROUP (sham, left vs. right DLPFC stimulation) as a between-subject factor revealed no group differences in any sessions $(p s>.11)$.
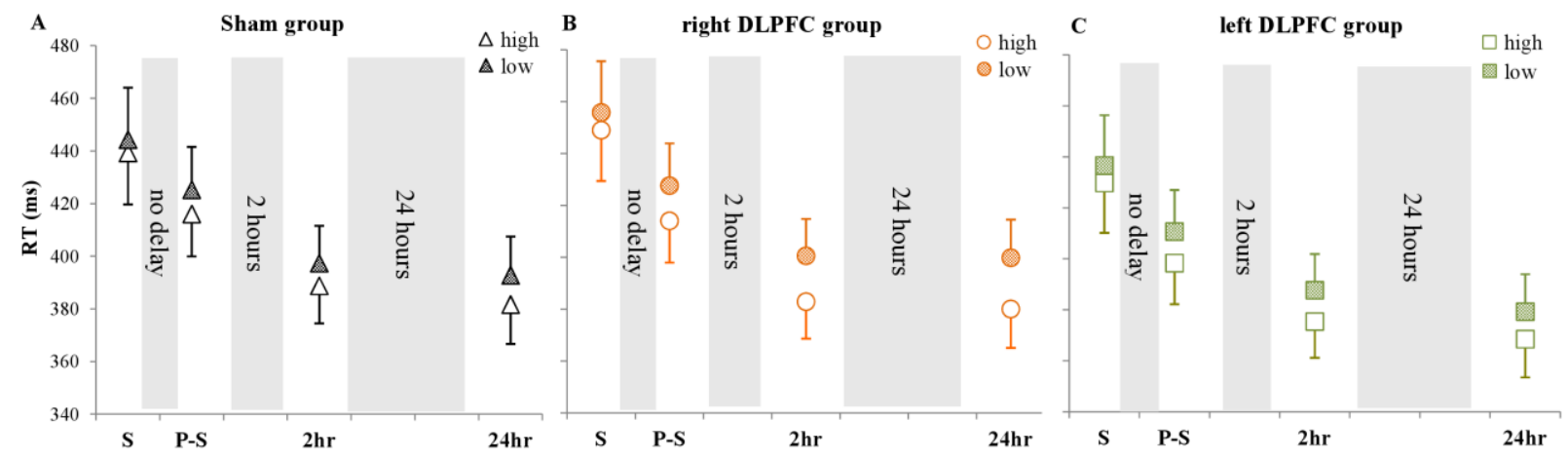
Figure 3. Raw reaction times (RTs) for high- and low-frequency triplets, for the sham (A), right DLPFC (B) and left DLPFC groups (C). S - Stimulation Phase, P-S - Post-Stimulation Phase, 2-hr and 24-hr Retention Phases. Error bars indicate Standard Error of the Mean (SEM).

\section{Discussion}

In our study, anodal tDCS over the right or left DLPFC was applied during a probabilistic sequence learning task in order to investigate the causal role of the targeted brain areas and the related networks in statistical learning and its consolidation. To our knowledge, this is the first study using tDCS over the DLPFCs to investigate consolidation of a sequence learning task with statistical features. Learning performance was assessed in the Stimulation, Post-Stimulation, 2and 24-hour Retention Phases (Fig. 1C). We found overall better learning performance of the right DLPFC group compared to the sham (control) group. When analyzing the sessions separately, there were no group differences in learning in the Stimulation and Post-Stimulation Phases. In contrast, the right DLPFC group exhibited a statistical learning advantage in the 2-hrand 24-hr-Retention Phases (Fig 2). We failed to find any effect of the left DLPFC stimulation.

Previous studies have demonstrated in split-brain and frontal lobe-lesioned patients that the right hemisphere, especially the right PFC has an advantage in learning statistical features of the environment $\left[\begin{array}{ll}7 & 13\end{array}\right]$. Here we used tDCS to directly manipulate the involvement of the targeted brain regions and the related networks in statistical learning. Our results partly complement the findings of those previous studies, since a better learning performance was observed after the right DLPFC stimulation compared to sham stimulation and by the last session also compared to the left DLPFC stimulation.

No effect of stimulation in the learning/stimulation phase might be due to the fact that we investigated a healthy, young population, while former studies primarily focused on patient populations [7 13]. In a healthy, young population, tDCS might cause a smaller change in task 
performance compared to the effect of a lesion in patients; the former is a short-term manipulation of the activity in the targeted brain regions and the related networks, while the latter could lead to an irreversible loss of a function subserved by the affected brain areas. In line with this argument, Friederici et al. [25] found an effect of left cathodal PFC stimulation on statistical language learning in healthy, young adults only in the testing session. One possible explanation is that the stimulation can lead to qualitative (different strategies, leading to more effective learning) or quantitative (stronger learning) differences in the learning/stimulation phase but these effects could not be revealed by behavioral measures. Future studies need to address this question using brain imaging or other sensitive methods to explore the effect of the stimulation on neural level during the learning phase.

Besides focusing on a healthy, young population, our study also went beyond the previous ones in that we examined the role of the right vs. left PFC not only in statistical learning itself, but also in the consolidation of the acquired information. Here we demonstrated that increased right PFC excitability results in a higher retention level compared to the sham condition both in the 2-hr and 24-hr Retention Phases, and also compared to the left PFC stimulation in the 24-hr Retention Phase. These findings highlight the role of the right PFC (and the right fronto-striatal network in general) in the stabilization of the representation of statistical regularities. Our findings are in line with the previously mentioned statistical language learning study of Friederici et al. [25] in which the cathodal stimulation of the left PFC during the learning phase resulted in a better performance in the testing phase. In our study we observed similar results with excitatory (anodal) stimulation of the right PFC instead of an inhibitory (cathodal) stimulation of the left PFC. Taken these findings together, a clear division of labor between right and left hemispheres can be observed in statistical learning mechanisms. 
Using a deterministic sequence learning task that has less prominent statistical features, Galea et al. [26] reported offline improvement following a "virtual lesion" of the right DLPFC. They used continuous theta-burst transcranial magnetic stimulation (cTBS) to either the right or the left DLPFC after the training. The cTBS has been shown to have an inhibiting effect on the stimulated area, resulting in a reversible, short-term functional lesion [27]. In contrast, the anodal tDCS used in our study has been shown to increase the excitability of the stimulated area, leading to higher neural plasticity. The seemingly surprising result that, compared to the left DLPFC, both the inhibition and excitation of the right DLPFC could lead to a superior performance after a retention period could be explained by the fact that we used the tDCS during the learning phase, which may affect the learning itself, while the stimulation in Galea et al.'s study [26] interfered with the consolidation of the previously acquired knowledge but not the acquisition itself. These results suggest that increased excitability of the right DLPFC in the acquisition phase might lead to a better learning performance after a retention period, while increased excitability of the right DLPFC after the acquisition phase might result in interference effects in the retention/consolidation of the acquired knowledge. Nevertheless, it is important to highlight that the tasks used in these two studies are quite different in nature (deterministic vs. probabilistic sequence learning) which can also lead to different effects of the stimulation. Future studies need to clarify this issue, directly manipulating both the direction (excitation vs. inhibition), type of the task (deterministic vs. probabilistic) and the timing of the stimulation (during or after the training).

Concerning the mechanisms of the effect of stimulation, on the cellular level anodal tDCS has been shown to cause membrane hypopolarization, leading to enhanced cortical excitability [28 29]. In addition, pharmacological studies suggest that the after-effects of the 
stimulation are N-methyl-D-aspartate (NMDA) receptor-dependent [30], sharing some similarities with long-term potentiation (LTP), which resemble neuroplastic alterations thought to underlie learning and memory formation [31 32]. On the network level, both imaging and modeling studies have been demonstrated that the stimulation affects not only the targeted brain region but also the interconnected areas [ [ 313334 . For instance, studies using resting state fMRI simultaneously with anodal tDCS over the right or left DLPFC showed hemisphere-specific increased synchronous activity and connectivity between lateral frontal and parietal areas [22 35]. Our results are in line with these findings showing a tDCS-induced hemisphere-specific modulation of statistical learning measured by a probabilistic sequence learning task. As a further step, future studies are needed to employ fMRI during the behavioral task and simultaneous stimulation, not only in resting state, in order to determine the effect of stimulation on the brain networks specifically involved in that particular task.

Previous research suggested that the effect of tDCS might be more general, facilitating reconfiguration of functional brain networks to address upcoming cognitive demands by, for example, increasing the temporal synchrony in the attention networks [22]. To partly address this concern, we administered a sleepiness scale to monitor changes in subjective alertness before and after the stimulation. The real and sham stimulation groups did not differ in subjective sleepiness neither after the stimulation, nor later in the retention phases. Our results indicate that the effect of stimulation is rather task-specific than general (e.g., alertness change). In addition, our finding that the stimulation of the left and right PFC led to different behavioral outcomes despite the similar level of alertness throughout the whole experiment also supports this notion.

Another important question is how long does the effect of stimulation last. Previous studies primarily investigated the after-effects of motor cortex stimulation and found that a 10- 
minute long tDCS induces neuroplastic changes up to 1 hour at least [36]. To our knowledge, the after-effects of tDCS on DLPFC have not yet been investigated in functional neuroimaging studies. Our results suggest that, on a behavioral level, the stimulation might have a delayed rather than an online effect. Nevertheless, studies examining various domains, such as working memory [20 37], short-term verbal learning [38] or planning [39], showed immediate improvements after anodal tDCS applied over the DLPFC. Future studies need to identify those specific cognitive processes which can immediately benefit from the stimulation versus those, which show delayed effects.

Taken together, we found hemispheric differences in the role of the PFCs in statistical learning and its consolidation with a probabilistic sequence learning task in healthy, young adults. In line with previous research [7], an advantage of the right PFC was obtained in picking up statistical regularities. As the involvement of the fronto-striatal network in statistical learning is well demonstrated, we believe that the boosting effect of the right PFC stimulation on learning processes is due to a greater excitability of the related fronto-striatal network, and not only of the PFC itself. In conclusion, our findings corroborate the previous ones showing hemispheric asymmetry with right hemisphere advantage in statistical learning and its consolidation, which can lead to development of new, more targeted treatment methods for learning and memory disorders.

\section{Acknowledgements}

Thanks to Russell A. Poldrack for the comments of the previous version of the manuscript. Thanks also to the members of the Imaging Research Center at University of Texas, Austin for numerous illuminating discussions. We thank Lena Sarp, Lauren Haag, Christin Fritsche, Maike 
Pelgrim for data collection, and Clare A. Tucker for proofreading. This research was supported by the Hungarian Science Foundation (OTKA NF 105878), the Hungarian-German Scientific Exchange Program (DAAD-MÖB) Grant number 29775 (2012-2013), and Janos Bolyai Research Fellowship of the Hungarian Academy of Sciences (KJ).

\section{References}

1. Daw ND, Niv Y, Dayan P. Uncertainty-based competition between prefrontal and dorsolateral striatal systems for behavioral control. Nature Neuroscience 2005;8(12):1704-11

2. Janacsek K, Fiser J, Nemeth D. The best time to acquire new skills: age-related differences in implicit sequence learning across the human lifespan. Developmental Science 2012;15(4):496-505

3. Orban G, Fiser J, Aslin RN, et al. Bayesian learning of visual chunks by human observers. Proceedings of the National Academy of Sciences 2008;105(7):2745-50

4. Fiser J, Berkes P, Orbán G, et al. Statistically optimal perception and learning: from behavior to neural representations. Trends in Cognitive Sciences;14(3):119-30

5. Nemeth D, Janacsek K, Fiser J. Age-dependent and coordinated shift in performance between implicit and explicit skill learning. Frontiers in Computational Neuroscience 2013;7 doi: 10.3389/fncom.2013.00147[published Online First: Epub Date]|.

6. Poldrack RA, Clark J, Pare-Blagoev EJ, et al. Interactive memory systems in the human brain. Nature 2001;414(6863):546-50

7. Roser ME, Fiser J, Aslin RN, et al. Right hemisphere dominance in visual statistical learning. Journal of cognitive neuroscience 2011;23(5):1088-99 
8. Fletcher PC, Zafiris $\mathrm{O}$, Frith $\mathrm{CD}$, et al. On the benefits of not trying: brain activity and connectivity reflecting the interactions of explicit and implicit sequence learning. Cerebral Cortex 2005;15(7):1002-15

9. Bennett IJ, Madden DJ, Vaidya CJ, et al. White matter integrity correlates of implicit sequence learning in healthy aging. Neurobiology of Aging 2011;32(12):2317.e1-17.e12

10. Stillman CM, Gordon EM, Simon JR, et al. Caudate resting connectivity predicts implicit probabilistic sequence learning. Brain connectivity 2013;3(6):601-10

11. Simon JR, Vaidya CJ, Howard JHJ, et al. The effects of aging on the neural basis of implicit associative learning in a probabilistic triplets learning task. Journal of Cognitive Neuroscience 2012;24(2):451-63

12. Gazzaniga MS. Principles of human brain organization derived from split-brain studies. Neuron 1995;14(2):217-28

13. Wolford G, Miller MB, Gazzaniga M. The left hemisphere's role in hypothesis formation. The Journal of Neuroscience 2000;20(6):RC64.

14. Howard JH, Jr., Howard DV. Age differences in implicit learning of higher-order dependencies in serial patterns. Psychology and Aging 1997;12(4):634-56

15. Song S, Howard JH, Jr., Howard DV. Sleep does not benefit probabilistic motor sequence learning. Journal of Neuroscience 2007;27(46):12475-83

16. Nemeth D, Janacsek K, Londe Z, et al. Sleep has no critical role in implicit motor sequence learning in young and old adults. Experimental Brain Research 2010;201(2):351-58 doi: 10.1007/s00221-009-2024-x[published Online First: Epub Date]|. 
17. Nemeth D, Janacsek K, Polner B, et al. Boosting Human Learning by Hypnosis. Cerebral Cortex 2013;23(4):801-05 doi: doi:10.1093/cercor/bhs068[published Online First: Epub Date]|.

18. Ambrus GG, Zimmer M, Kincses ZT, et al. The enhancement of cortical excitability over the DLPFC before and during training impairs categorization in the prototype distortion task. Neuropsychologia 2011;49(7):1974-80

19. Marshall L, Mölle M, Hallschmid M, et al. Transcranial direct current stimulation during sleep improves declarative memory. The Journal of neuroscience 2004;24(44):9985-92

20. Marshall L, Mölle M, Siebner H, et al. Bifrontal transcranial direct current stimulation slows reaction time in a working memory task. BMC neuroscience 2005;6(1):23

21. Herwig U, Satrapi P, Schönfeldt-Lecuona C. Using the international 10-20 EEG system for positioning of transcranial magnetic stimulation. Brain topography 2003;16(2):95-99

22. Peña-Gómez C, Sala-Lonch R, Junqué C, et al. Modulation of large-scale brain networks by transcranial direct current stimulation evidenced by resting-state functional MRI. Brain stimulation 2012;5(3):252-63

23. Hoddes E, Dement W, Zarcone V. The development and use of the Stanford Sleepiness Scale (SSS). Psychophysiology 1972;9(150):431-36

24. Howard DV, Howard JH, Jr., Japikse K, et al. Implicit sequence learning: effects of level of structure, adult age, and extended practice. Psychology and Aging 2004;19(1):79-92

25. Friederici AD, Mueller JL, Sehm B, et al. Language learning without control: the role of the PFC. Journal of cognitive neuroscience 2013;25(5):814-21 
26. Galea JM, Albert NB, Ditye T, et al. Disruption of the dorsolateral prefrontal cortex facilitates the consolidation of procedural skills. Journal of cognitive neuroscience 2010;22(6):1158-64

27. Huang Y-Z, Edwards MJ, Rounis E, et al. Theta burst stimulation of the human motor cortex. Neuron 2005;45(2):201-06

28. Bindman LJ, Lippold O, Redfearn J. The action of brief polarizing currents on the cerebral cortex of the rat (1) during current flow and (2) in the production of long-lasting aftereffects. The Journal of physiology 1964;172(3):369-82

29. Creutzfeldt OD, Fromm GH, Kapp H. Influence of transcortical dc currents on cortical neuronal activity. Experimental neurology 1962;5(6):436-52

30. Liebetanz D, Nitsche M, Tergau F, et al. Pharmacological approach to the mechanisms of transcranial DC-stimulation-induced after-effects of human motor cortex excitability. Brain 2002;125(10):2238-47

31. Polanía R, Paulus W, Antal A, et al. Introducing graph theory to track for neuroplastic alterations in the resting human brain: a transcranial direct current stimulation study. Neuroimage 2011;54(3):2287-96

32. Rioult-Pedotti M-S, Friedman D, Donoghue JP. Learning-induced LTP in neocortex. Science 2000;290(5491):533-36

33. Antal A, Polania R, Schmidt-Samoa C, et al. Transcranial direct current stimulation over the primary motor cortex during fMRI. Neuroimage 2011;55(2):590-96

34. Kwon YH, Ko M-H, Ahn SH, et al. Primary motor cortex activation by transcranial direct current stimulation in the human brain. Neuroscience letters 2008;435(1):56-59 
35. Keeser D, Meindl T, Bor J, et al. Prefrontal transcranial direct current stimulation changes connectivity of resting-state networks during fMRI. The Journal of Neuroscience 2011;31(43):15284-93

36. Nitsche M, Niehaus L, Hoffmann K, et al. MRI study of human brain exposed to weak direct current stimulation of the frontal cortex. Clinical Neurophysiology 2004;115(10):2419-23

37. Zaehle T, Sandmann P, Thorne J, et al. Transcranial direct current stimulation of the prefrontal cortex modulates working memory performance: combined behavioural and electrophysiological evidence. BMC neuroscience 2011;12(1):2

38. Elmer S, Burkard M, Renz B, et al. Direct current induced short-term modulation of the left dorsolateral prefrontal cortex while learning auditory presented nouns. Behavioral and Brain Functions 2009;5(1):29

39. Dockery CA, Hueckel-Weng R, Birbaumer N, et al. Enhancement of planning ability by transcranial direct current stimulation. The Journal of Neuroscience 2009;29(22):7271-77 